\title{
A COVID-19 Case Complicated by Candida dublinien- sis and Klebsiella pneumoniae-Carbapenem-Resistant Enterobacteriaceae
}

\author{
Harith Alataby ${ }^{\mathrm{a}}$, Francis Atemnkeng, ${ }^{\mathrm{a}, \mathrm{b}}$, Sandeep S. Bains ${ }^{\mathrm{a}}$, Foma M. Kenne ${ }^{\mathrm{a}}$, \\ Keith Diaz ${ }^{\mathrm{a}}$, Jay Nfonoyim ${ }^{\mathrm{a}}$
}

\begin{abstract}
There has been increasing evidence of co-infections with coronavirus disease 2019 (COVID-19) pneumonia, which increases the severity of the disease. Organisms such as Klebsiella pneumoniae and Streptococcus pneumoniae have been previously isolated. We present a case of a COVID-19 patient treated with baricitinib and dexamethasone who later developed Klebsiella pneumoniae-carbapenem-resistant Enterobacteriaceae (CRE) and Candida dubliniensis bloodstream infections, treated with meropenem/vaborbactam and micafungin, respectively. These infections are exceedingly rare and are mostly reported in immunosuppressed patients. The finding of these bloodstream infections raises concerns on the cause of immunosuppression in this patient infected with severe acute respiratory syndrome coronavirus 2 (SARS-CoV-2) treated with baricitinib and dexamethasone. There has been no report so far of COVID-19 associated with these co-infections.
\end{abstract}

Keywords: COVID-19; Bacteremia; Candidemia; Baricitinib; Dexamethasone

\section{Introduction}

Since the start of the coronavirus disease 2019 (COVID-19) pandemic, there has been an increasing need for treatments that can reduce the morbidity, mortality, and length of stay of patients both in the intensive care unit (ICU) and hospital in general [1]. With so many unknown variables involved, it is very unpredictable what further complications are expected, including the management of patients with immunomodula-

Manuscript submitted September 21, 2020, accepted October 3, 2020

Published online October 21, 2020

aDepartment of Medicine, Richmond University Medical Center, Staten Island, NY 10310, USA

${ }^{b}$ Corresponding Author: Francis Atemnkeng, Department of Medicine, Richmond University Medical Center, Staten Island, NY 10310, USA.

Email: fatemnkeng@rumcsi.org

doi: https://doi.org/10.14740/jmc3588 tory treatments and steroids [2-4]. The difficulty, in this case, increased due to the subsequent bacteremia and fungemia that were caused by rare pathogens. These pathogens are Klebsiella pneumoniae-carbapenem-resistant Enterobacteriaceae (CRE) and Candida dubliniensis. Klebsiella pneumoniae-CRE is rapidly emerging around the world with a high case fatality ratio because of the minimal treatment options available [5-7]. Candida dubliniensis, on the other hand, has also been reported mostly in immunocompromised patients with oropharyngeal infections and rarely in the bloodstream $[8,9]$. In this case report, we present a patient who tested positive for COVID-19, and treated with immunomodulatory therapy, including baricitinib and dexamethasone, who was later found to have Candida dubliniensis and Klebsiella pneumoniae-CRE in the bloodstream. No other case has been reported so far.

\section{Case Report}

A 67-year-old morbidly obese and quadriplegic man who has a very extensive past medical history including chronic obstructive pulmonary disease (COPD), diabetes mellitus, hypertension, chronic kidney disease, peripheral vascular disease, and hepatitis $\mathrm{C}$, who was sent from a skilled nursing facility with respiratory distress, which started about 1 day before admission. On arrival in the emergency room (ER), he had a pulse of 122 , respiratory rate of 44 , blood pressure of $95 / 45 \mathrm{~mm} \mathrm{Hg}$, temperature of $36.9^{\circ} \mathrm{C}$, and saturating at $78 \%$ in room air. On physical exam, his Glasgow coma score was 7 , responding only to painful stimuli, he was in severe respiratory distress, using accessory muscles, and had bilateral crackles on auscultation. He was subsequently intubated and placed on mechanical ventilation, and then transferred to the medical ICU. A COVID-19 RNA polymerase chain reaction (PCR) test was done, which came back positive, and he was treated with the standard care at the time for COVID-19 (hydroxychloroquine, azithromycin, vitamin $\mathrm{C}$, and zinc). Two days later, the patient was extubated and placed on a $50 \%$ Venturi mask. His evolution was marked by the persistence of hypotension and worsening of respiratory distress with increasing oxygen requirements and worsening imaging exams. Due to the worsening of the respiratory status of the patient and increasing reports on the benefits of dexamethasone and 
Table 1. Evolution of Labs From the Day of Admission to Discharge

\begin{tabular}{|c|c|c|c|c|c|c|c|c|c|c|}
\hline & Day 1 & Day 16 & Day 17 & Day 18 & Day 19 & Day 20 & Day 21 & Day 22 & Day 24 & Day 34 \\
\hline WBC $(\mathrm{k} / \mu \mathrm{L})$ & 8.7 & 9.2 & 18.1 & 20.0 & 32.3 & 35.2 & 32.1 & 24.8 & 10.2 & 5.3 \\
\hline $\mathrm{RBC}(\mathrm{m} / \mu \mathrm{L})$ & 3.39 & 3.79 & 4.06 & 3.58 & 3.52 & 3.32 & 3.62 & 3.62 & 4.06 & 3.48 \\
\hline Hct $(\%)$ & 31.5 & 36.3 & 38.7 & 34.4 & 34.1 & 31.3 & 35.1 & 35.1 & 38.6 & 32.5 \\
\hline MCV (fL) & 92.8 & 95.8 & 95.4 & 96.2 & 97 & 94.3 & 97.1 & 96.8 & 94.9 & 93.4 \\
\hline RDW (\%) & 17.7 & 18.4 & 18.4 & 20.0 & 19.9 & 19.8 & 19.8 & 19.8 & 18.9 & 19.8 \\
\hline Platelet $(\mathrm{k} / \mu \mathrm{L})$ & 299 & 413 & 413 & 304 & 225 & 203 & 186 & 179 & 176 & 145 \\
\hline
\end{tabular}

WBC: white blood count; RBC: red blood count; Hgb: hemoglobin; Hct: hematocrit; MCV: mean corpuscular volume; $\mathrm{MCH}$ : mean corpuscular hemoglobin; MCHC: mean corpuscular hemoglobin concentration; RDW: red cell distribution width.

baricitinib, he was given dexamethasone $15 \mathrm{mg}$ intravenously (IV) for 5 days and baricitinib $2 \mathrm{mg}$ for 5 days. On day 17 of admission, 4 days after completion of baricitinib and dexamethasone, the patient became hypotensive, tachycardic with a blood pressure of 91/52, and heart rate of 115 while on continuous positive airway pressure ventilation (CPAP) saturating at $96 \%$. We noticed a marked increase in his white blood cell count from 9.2 to $18.1 \mathrm{k} / \mu \mathrm{L}$ on this day (Table 1 ). Blood and urine cultures were done, and he was started on IV fluids and empirical antibiotics, including vancomycin and piperacillin/tazobactam.

Blood cultures grew Klebsiella pneumoniae-CRE (sensitivity patterns are shown in Table 2). Because meropenem showed an intermediate sensitivity, antibiotics were switched to meropenem/vaborbactam. Subsequent blood cultures still grew Klebsiella pneumoniae-CRE on three consecutive cultures drawn on three different days. Seven days after starting meropenem/vaborbactam, the blood cultures became positive to Candida dubliniensis twice on two consecutive blood cul- tures drawn on two different days. He was then started on IV micafungin $100 \mathrm{mg}$, which he received for 10 days and then switched to oral fluconazole for 14 days. Meropenem/ vaborbactam was discontinued 14 days after the first negative blood culture. His repeat blood cultures and COVID-19 were negative and he was discharged back to his skilled nursing facility.

\section{Discussion}

Severe acute respiratory syndrome coronavirus 2 (SARSCoV-2) is an enveloped, single-stranded positive-sense RNA virus that affects human cells by spike glycoprotein binding to its cellular receptor, angiotensin-converting enzyme 2 (ACE2) [10]. With over 20,000,000 confirmed cases and 736,000 deaths, COVID-19 is wreaking havoc across the globe. The incubation period of the virus ranges from 3 to 21 days [11], after which most patients present with either mild non-specific

Table 2. Sensitivity Profile of Klebsiella pneumoniae-CRE Isolated in the Patient

\begin{tabular}{lll}
\hline Antibiotic & Minimal inhibitory concentration (mg/L) & Susceptibilities \\
\hline Ampicillin & $\geq 32$ & $\mathrm{R}$ \\
Ampicillin/sulbactam & $\geq 32$ & $\mathrm{R}$ \\
Aztreonam & $\geq 64$ & $\mathrm{R}$ \\
Cefazolin & $\geq 64$ & $\mathrm{R}$ \\
Cefepime & $\geq 64$ & $\mathrm{R}$ \\
Ceftazidime & $\geq 64$ & $\mathrm{R}$ \\
Ceftriaxone & $\geq 64$ & $\mathrm{R}$ \\
Gentamycin & $\geq 16$ & $\mathrm{R}$ \\
Levofloxacin & $\geq 8$ & $\mathrm{R}$ \\
Meropenem & 8 & $\mathrm{I}$ \\
Piperacillin/tazobactam & $\mathrm{R}$ \\
Tobramycin & $\geq 128$ & $\mathrm{R}$ \\
Trimethoprim/sulfamethoxazole & $\mathrm{R}$ \\
\hline
\end{tabular}

R: resistant; I: intermediate; CRE: carbapenem-resistant Enterobacteriaceae. 
symptoms or severe pneumonia with multiorgan dysfunction [12-14].

With the surge of COVID-19 patients in March 2020, New York, the epitome of the disease at that time, also observed a surge in blood cultures [15]. However, this surge of blood cultures showed that those infected with COVID-19 had lower rates of bacteremia. But respiratory co-infections were high and often a significant cause of mortality [16]. Commonly isolated respiratory co-infection organisms were Streptococcus pneumoniae, Klebsiella pneumoniae, Mycoplasma pneumoniae, Pseudomonas aeruginosa, and Hemophilus influenzae. Due to the increased mortality with concomitant bacterial infections with the previous influenza pandemic, a systematic review found about 7\% of COVID-19 patients had co-infections and mostly patients in the ICU [17]. Our case had a positive blood culture to a very resistant bacteria called Klebsiella pneumoniae-CRE and Candida dubliniensis, which is the only case associated with COVID-19 reported so far. CRE in particular was labeled an "urgent threat" in the 2013 and 2019 antibiotic resistance threats in the USA reports by the US Centers for Disease Control and Prevention (CDC) $[18,19]$.

Candida dubliniensis is also a very rare organism, mostly found in oropharyngeal infections in immunosuppressed individuals $[8,9]$. The presence of this organism in the blood culture of our patient suggests underlying immunosuppression, which needs to be explored. This immunosuppression could be due to the use of baricitinib and dexamethasone in our patient [20], or it could suggest immunosuppression caused by COVID-19 not yet demonstrated.

With the increasing evidence of the presence of hyperinflammation and cytokine storm and high levels of IL-6 being associated with increased mortality in COVID-19 patients, treatment with immunomodulatory therapies like baricitinib and steroids like dexamethasone has been shown to reduce mortality $[3,4,21]$. The use of baricitinib in the treatment of rheumatoid arthritis was proven to cause immunosuppression; the most frequent infection associated with this was herpes zoster [20]. With the use of baricitinib and dexamethasone in combination in the management of COVID-19 patients, they tend to become more immunosuppressed, and therefore, susceptible to many infections.

Klebsiella pneumoniae-CRE has a very low sensitivity profile to most antibiotic categories and is very challenging to treat [22]. One drug combination that shows universal coverage against beta-lactamase-producing Enterobacteriaceae, including those with extensive drug resistance, is aztreonam and ceftazidime/avibactam [22]. However, our patient had resistance to aztreonam and ceftazidime, which made it even more challenging to treat. Even though it had intermediate sensitivity to meropenem alone, we used it in the combination of meropenem/vaborbactam as the only available choice of treatment. The high case fatality ratio of this organism is due to the very resistant properties.

It is very rare to have fungemia due to Candida dubliniensis without any immunosuppression. This is why, despite the short period of immunomodulatory treatment in our patient, the discovery of Candida dubliniensis in two consecutive blood cultures is most likely because of the relative im- munosuppression. For the treatment of Candida dubliniensis, treatment options vary per Infectious Disease Society guidelines. Currently, it is recommended to use echinocandin with fluconazole being an appropriate alternative [9]. However, some reports have shown resistance to fluconazole and must be taken into consideration [23]. Follow-up blood cultures should be taken until a negative result is achieved, and at this point, treatment should subside 2 weeks later. Our patient was treated with micafungin for 10 days, with repeat blood cultures which came back negative, and he was then discharged with an additional 2 weeks of oral fluconazole.

\section{Conclusions}

In this case, we were able to present a patient with multiple comorbidities infected with COVID-19 whom we treated with baricitinib and dexamethasone for 5 days, who developed Klebsiella pneumoniae-CRE and Candida dubliniensis in blood cultures. The use of immunomodulators might have caused relative immunosuppression, hence increasing the susceptibility of the patient developing rare bloodstream infections, therefore, increasing the complexity of the management of COVID-19.

\section{Acknowledgments}

None to declare.

\section{Financial Disclosure}

None to declare.

\section{Conflict of Interest}

None to declare.

\section{Informed Consent}

Not applicable.

\section{Author Contributions}

HA collected the data, guided the literature search, wrote the manuscript, and is the research guarantor. FA, SSB, FMK and $\mathrm{KD}$ helped with data collection and writing of the article. JN reviewed and supervised the study.

\section{Data Availability}

The authors declare that data supporting the findings of this study are available within the article. 


\section{References}

1. Wang Y, Liao B, Guo Y, Li F, Lei C, Zhang F, Cai W, et al. Clinical characteristics of patients infected with the novel 2019 coronavirus (SARS-Cov-2) in Guangzhou, China. Open Forum Infect Dis. 2020;7(6):ofaa187.

2. Cantini F, Niccoli L, Matarrese D, Nicastri E, Stobbione P, Goletti D. Baricitinib therapy in COVID-19: A pilot study on safety and clinical impact. J Infect. 2020;81(2):318356.

3. Cingolani A, Tummolo AM, Montemurro G, Gremese E, Larosa L, Cipriani MC, Pasciuto G, et al. Baricitinib as rescue therapy in a patient with COVID-19 with no complete response to sarilumab. Infection. 2020;48(5):767-771.

4. Titanji BK, Farley MM, Mehta A, Connor-Schuler R, Moanna A, Cribbs SK, O'Shea J, et al. Use of Baricitinib in Patients with Moderate and Severe COVID-19. Clin Infect Dis. 2020.

5. Cuzon G, Ouanich J, Gondret R, Naas T, Nordmann P. Outbreak of OXA-48-positive carbapenem-resistant Klebsiella pneumoniae isolates in France. Antimicrob Agents Chemother. 2011;55(5):2420-2423.

6. Carrer A, Poirel L, Yilmaz M, Akan OA, Feriha C, Cuzon G, Matar G, et al. Spread of OXA-48-encoding plasmid in Turkey and beyond. Antimicrob Agents Chemother. 2010;54(3):1369-1373.

7. Cuzon G, Naas T, Bogaerts P, Glupczynski Y, Huang TD, Nordmann P. Plasmid-encoded carbapenem-hydrolyzing beta-lactamase OXA-48 in an imipenem-susceptible Klebsiella pneumoniae strain from Belgium. Antimicrob Agents Chemother. 2008;52(9):3463-3464.

8. Martinez M, Lopez-Ribot JL, Kirkpatrick WR, Coco BJ, Bachmann SP, Patterson TF. Replacement of Candida albicans with $\mathrm{C}$. dubliniensis in human immunodeficiency virus-infected patients with oropharyngeal candidiasis treated with fluconazole. J Clin Microbiol. 2002;40(9):3135-3139.

9. Sullivan D, Coleman D. Candida dubliniensis: characteristics and identification. J Clin Microbiol. 1998;36(2):329334.

10. Shereen MA, Khan S, Kazmi A, Bashir N, Siddique R. COVID-19 infection: Origin, transmission, and characteristics of human coronaviruses. J Adv Res. 2020;24:9198.

11. Qin J, You C, Lin Q, Hu T, Yu S, Zhou XH. Estimation of incubation period distribution of COVID-19 using disease onset forward time: a novel cross-sectional and forward follow-up study. medRxiv. 2020.
12. Liu J, Cui M, Yang T, Yao P. Correlation between gastrointestinal symptoms and disease severity in patients with COVID-19: a systematic review and meta-analysis. BMJ Open Gastroenterol. 2020;7(1):e000437.

13. Ge H, Wang X, Yuan X, Xiao G, Wang C, Deng T, Yuan $\mathrm{Q}$, et al. The epidemiology and clinical information about COVID-19. Eur J Clin Microbiol Infect Dis. 2020;39(6):1011-1019.

14. Huang X, Wei F, Hu L, Wen L, Chen K. Epidemiology and clinical characteristics of COVID-19. Arch Iran Med. 2020;23(4):268-271.

15. Sepulveda J, Westblade LF, Whittier S, Satlin MJ, Greendyke WG, Aaron JG, Zucker J, et al. Bacteremia and blood culture utilization during COVID-19 surge in New York City. J Clin Microbiol. 2020;58(8).

16. Zhu X, Ge Y, Wu T, Zhao K, Chen Y, Wu B, Zhu F, et al. Co-infection with respiratory pathogens among COVID-2019 cases. Virus Res. 2020;285:198005.

17. Lansbury L, Lim B, Baskaran V, Lim WS. Co-infections in people with COVID-19: a systematic review and metaanalysis. J Infect. 2020;81(2):266-275.

18. Logan LK, Weinstein RA. The epidemiology of carbapenem-resistant enterobacteriaceae: the impact and evolution of a global menace. J Infect Dis. 2017;215(suppl_1):S28S36.

19. Livorsi DJ, Chorazy ML, Schweizer ML, Balkenende EC, Blevins AE, Nair R, Samore MH, et al. A systematic review of the epidemiology of carbapenem-resistant Enterobacteriaceae in the United States. Antimicrob Resist Infect Control. 2018;7:55.

20. Taylor PC, Keystone EC, van der Heijde D, Weinblatt ME, Del Carmen Morales L, Reyes Gonzaga J, Yakushin $\mathrm{S}$, et al. Baricitinib versus placebo or adalimumab in rheumatoid arthritis. N Engl J Med. 2017;376(7):652-662.

21. Recovery Collaborative Group, Horby P, Lim WS, Emberson JR, Mafham M, Bell JL, Linsell L, et al. Dexamethasone in hospitalized patients with COVID-19 - preliminary report. N Engl J Med. 2020.

22. Krapp F, Ozer EA, Qi C, Hauser AR. Case report of an extensively drug-resistant klebsiella pneumoniae infection with genomic characterization of the strain and review of similar cases in the United States. Open Forum Infect Dis. 2018;5(5):ofy074.

23. Pappas PG, Kauffman CA, Andes DR, Clancy CJ, Marr KA, Ostrosky-Zeichner L, Reboli AC, et al. Clinical practice guideline for the management of candidiasis: 2016 update by the infectious diseases society of America. Clin Infect Dis. 2016;62(4):e1-50. 